\title{
Using geometric control and chaotic synchronization to estimate an unknown model parameter
}

\author{
Ubiratan S. Freitas, ${ }^{1, *}$ Elbert E. N. Macau, ${ }^{1, \dagger}$ and Celso Grebogi ${ }^{2}$ \\ ${ }^{1}$ Laboratório Associado de Computação e Matemática Aplicada, Instituto Nacional de Pesquisas Espaciais, \\ Caixa Postal 515, 12227-010, São José dos Campos, São Paulo, Brazil \\ ${ }^{2}$ Instituto de Física, Universidade de São Paulo, 05315-970 São Paulo, São Paulo, Brazil
}

(Received 1 October 2004; published 27 April 2005)

\begin{abstract}
We present a new parameter estimation procedure for nonlinear systems. Such technique is based on the synchronization between the model and the system whose unknown parameter is wanted. Synchronization is accomplished by controlling the model to make it follow the system. We use geometric nonlinear control techniques to design the control system. These techniques allow us to derive sufficient conditions for synchronization and hence for proper parameter estimation. As an example, this procedure is used to estimate a parameter of an example serving as a model.
\end{abstract}

DOI: 10.1103/PhysRevE.71.047203

PACS number(s): 05.45.Gg, 05.45.Xt

Since 1990 [1,2], chaos synchronization has been a subject of intense study and is now considered to be a fundamental mechanism behind a variety of behaviors in nature [3]. As a consequence of the intense research effort, a multiplicity of applications was and is being realized in a variety of areas, e.g., in optics [4], communication [5], and neural networks [6]. In the majority of applications, we have the scenario where a chaotic system, called the sender or master, generates a signal which is sent over a channel to a receiver. The receiver (or slave) uses this signal to synchronize itself to the master.

Behind this scenario, there is the assumption that the parameters in both sender and receiver systems are identical. However, in a real situation, this assumption is unrealistic. For example, two electronic components are never identical, even if they came from the same assembly line. As a consequence, parameters on different systems are unequal with probability one. This reality has severe consequences in the context of chaotic synchronization: progressive parameter mismatch may successively imply a large burst of desynchronization (bubbling transition), blowout bifurcation, and lag synchronization [7], among other phenomena.

In this work we devise a synchronization strategy that allows us to estimate an unknown parameter of the master system. Other authors have also used synchronization to estimate unknown parameters [8-10]. Their work, however, does not provide sufficient conditions for a general coupling between master and slave to yield synchronization and, hence, a proper parameter estimation. Our approach, on the other hand, provides a straightforward procedure that, given a master system, will check if the strategy is applicable and, if so, will design a coupling that insures synchronization. Such improvement is possible because we base our strategy on geometric control $[11,12]$. Presently, our strategy can estimate a single unknown parameter of the sender, but this could be easily extended to many parameters.

The main idea in our approach is to treat synchronization as a control problem [13]. For a recent application of this

\footnotetext{
*Electronic address: ubiratan@lac.inpe.br

${ }^{\dagger}$ Electronic address: elbert@lac.inpe.br
}

concept to chaotic systems with uncertainties see [14]. In our approach, we conceive the receiver as a system to be controlled. Its unknown parameter is considered as the control input. The controller, which is designed based on the geometric control theory, dynamically adjusts this unknown parameter to make the slave follow a desired trajectory. Since synchronization is our goal, the desired trajectory is the one generated by the master system. The controller then acts as the coupling between the master and the slave systems. We show that the control action, i.e., the unknown parameter, equals the corresponding parameter of the master system, once synchronization is achieved. Actually, using these design techniques we can establish sufficient conditions for controllability, and hence for synchronizability and parameter recovery.

For concreteness, we consider two almost identical $n$-dimensional systems, system $A$ (the sender): $\dot{\boldsymbol{X}}=\boldsymbol{F}(\boldsymbol{X}, p)$ and system $B$ (the receiver): $\dot{\boldsymbol{Y}}=\boldsymbol{F}(\boldsymbol{Y}, u)$, where $\boldsymbol{X}, \boldsymbol{Y} \in \mathbb{R}^{n}$ are local coordinates for a smooth manifold $M, \boldsymbol{F}$ is a smooth function in its variables, $p$ for system $A$ is a fixed parameter value, and $u$ for system $B$ is an unknown and externally adjustable parameter. We consider that the two systems are initialized with different initial conditions, and the signal $\boldsymbol{X}$ is transmitted to the receiver (system $B$ ) so that this signal is available at all times at the receiver location. Our strategy is depicted in Fig. 1.

As can be seen from this picture, the controller uses the information provided by signals $\boldsymbol{X}$ and $\boldsymbol{Y}$ to instantly adjust the system $B$ parameter $u$ until this system is completely synchronized to the system $A$, i.e., $\boldsymbol{X}(t)=\boldsymbol{Y}(t)$ for $t>t_{s}$. Actually, the parameter $u$ is regarded as a control input for the

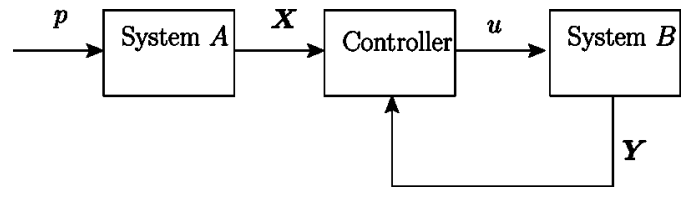

FIG. 1. The diagram of the synchronization strategy. The slave system is regarded as a system to be controlled. The controller acts on the parameter $p$ in order to make the slave to follow the master system. 
slave system. The controller has to correctly adjust this input for the synchronization to occur.

We assume that both systems can be written in a smooth affine form as follows:

$$
\text { system } A(\text { sender }): \dot{\boldsymbol{X}}=\boldsymbol{G}(\boldsymbol{X})+p \boldsymbol{H}(\boldsymbol{X})
$$

and

$$
\text { system } B(\text { receiver }): \dot{\boldsymbol{Y}}=\boldsymbol{G}(\boldsymbol{Y})+u \boldsymbol{H}(\boldsymbol{Y}),
$$

where $u$ is the control input. Let us define the synchronization error $\boldsymbol{E}(t)=\boldsymbol{X}(t)-\boldsymbol{Y}(t)$, and then the error dynamics is $\dot{\boldsymbol{E}}=\boldsymbol{G}(\boldsymbol{X})-\boldsymbol{G}(\boldsymbol{Y})+p \boldsymbol{H}(\boldsymbol{X})-u \boldsymbol{H}(\boldsymbol{Y})$. Now, suppose that synchronization is achieved. This implies that $\boldsymbol{E}(t)=\dot{\boldsymbol{E}}(t)=0$, for $t>t_{s}$. Thus, $\dot{\boldsymbol{E}}(t)$ at $\boldsymbol{E}=0$ is given by

$$
\dot{\boldsymbol{E}}=(p-u) \boldsymbol{H}(\boldsymbol{X}) .
$$

With the restriction $\dot{\boldsymbol{E}}=0$, i.e., both systems remain synchronized, we conclude that either $\boldsymbol{H}(\boldsymbol{X})=0$ for $t>t_{s}$ or $u=p$. We may discard the former because otherwise it would imply that our systems are independent of their parameters, which contradicts our assumptions. Then, we must conclude that, under these conditions, synchronization implies proper parameter estimation. So, in order to recover the unknown parameter, we must design a control system that synchronizes the receiver to the sender.

Such control system must act on a nonlinear system (the chaotic slave), through a specific input (the unknown parameter), following a reference signal that is not periodic nor has negligible amplitude (the master's chaotic trajectory). Moreover, the tracking error must be as small as possible. These are rather difficult requirements for a control system design and traditional linear techniques cannot be used. Any control design technique suitable to nonlinear systems could be used. We chose geometric control theory because it allows us to check if a nonlinear system is controllable and, if it is, provides a design method that will lead to a successful controller. In other words, geometric control theory gives us necessary and sufficient conditions for the controllability of a nonlinear system, and, if the system is controllable, a procedure to design a controller.

At this point, let us give some background on geometric control theory. Let $M$ be a smooth manifold of dimension $n$ and $(U, \varphi)=\left(U, x_{1}, \ldots, x_{n}\right)$ be a local coordinate chart for $M$. A smooth vector field $\boldsymbol{G}$ on $M$ assigns to every $q \in M$ a tangent vector $\boldsymbol{G}_{q} \in T_{q} M$. Given a smooth vector field $\boldsymbol{G}$ and a function $f: M \rightarrow \mathbb{R}$, the function $\boldsymbol{G}(f)(q): M \rightarrow \mathbb{R}$ is called the total derivative of $f$ along $\boldsymbol{G}$, or the Lie derivative of $f$ along $\boldsymbol{G}$ and is denoted as $\mathrm{L}_{\boldsymbol{G}} f$. If $\boldsymbol{G}$ is expressed in local coordinates as the vector $\left[x_{1}(q), x_{2}(q), \ldots, x_{n}(q)\right]^{\mathrm{T}}$, then we have

$$
\begin{aligned}
\mathrm{L}_{\boldsymbol{G}} f(q)=\boldsymbol{G}(f)(q) & =\sum_{i=1}^{n} \frac{\partial f}{\partial x_{i}}\left[x_{1}, x_{2}, \ldots, x_{n}\right] \boldsymbol{G}_{i}\left[x_{1}, x_{2}, \ldots, x_{n}\right] \\
& =\boldsymbol{\nabla} f \boldsymbol{G}(q) .
\end{aligned}
$$

For $\boldsymbol{G}$ and $\boldsymbol{H}$ any two smooth vector fields on $M$, we define a new vector field, denoted as $[\boldsymbol{G}, \boldsymbol{H}]$, or $\operatorname{ad}_{\boldsymbol{G}} \boldsymbol{H}$, called the Lie bracket of $\boldsymbol{G}$ and $\boldsymbol{H}$ by setting, in local coordinates,

$$
[G, H]=\nabla H G-\nabla G H,
$$

where $\boldsymbol{\nabla} \boldsymbol{G}$ is the Jacobian matrix of $\boldsymbol{G}$.

Our strategy can be divided in two steps. In the first one, we need to find a transformation pair as follows,

$$
\tilde{\boldsymbol{Y}}=\Phi(\boldsymbol{Y})
$$

and

$$
\widetilde{u}=\Psi(\boldsymbol{Y}, u),
$$

that transforms Eq. (2) to the following linear system:

$$
\dot{\tilde{y}_{i}}=\tilde{y}_{i+1}, \quad i=1, \ldots, n-1, \quad \dot{\tilde{y}}_{n}=\widetilde{u} .
$$

According to the geometric control theory $[11,15]$, this transformation is always possible wherever there exists an open region $\Omega \subset M$ so that the following conditions hold for all $q \in \Omega$ :

- the set of vector fields $\left\{\boldsymbol{H}, \operatorname{ad}_{G} \boldsymbol{H}, \ldots, \operatorname{ad}_{\boldsymbol{G}}^{n-1} \boldsymbol{H}\right\}$ is linearly independent, and

- the set of vector fields $\left\{\boldsymbol{H}, \operatorname{ad}_{\boldsymbol{G}} \boldsymbol{H}, \ldots, \mathrm{ad}_{\boldsymbol{G}}^{n-2} \boldsymbol{H}\right\}$ is involutive.

A set of vector fields is involutive if the Lie bracket of every two of its elements can be expressed as a linear combination of the vector fields in the set. If these two conditions hold, the transformations (6) and (7) exist and can be calculated as follows:

$$
\Phi(\boldsymbol{Y})=\left[\phi(\boldsymbol{Y}) \mathrm{L}_{\boldsymbol{G}} \phi(\boldsymbol{Y}) \cdots \mathrm{L}_{\boldsymbol{G}}^{n-1} \phi(\boldsymbol{Y})\right]^{\mathrm{T}},
$$

where $\phi(\cdot)$ is a solution of the system of partial differential equations

$$
\begin{gathered}
\mathrm{L}_{\boldsymbol{H}} \phi(\boldsymbol{Y})=0, \\
\mathrm{~L}_{\boldsymbol{H}} \mathrm{L}_{\boldsymbol{G}} \phi(\boldsymbol{Y})=0, \\
\vdots \\
\mathrm{L}_{\boldsymbol{H}} \mathrm{L}_{\boldsymbol{G}}^{n-2} \phi(\boldsymbol{Y})=0, \\
\mathrm{~L}_{\boldsymbol{H}} \mathrm{L}_{G}^{n-1} \phi(\boldsymbol{Y}) \neq 0 .
\end{gathered}
$$

Furthermore, the inverse input transformation $u=\Psi^{-1}(\boldsymbol{Y}, \widetilde{u})$ is given by

$$
\Psi^{-1}(\boldsymbol{Y}, \tilde{u})=\frac{\widetilde{u}-\alpha(\boldsymbol{Y})}{\beta(\boldsymbol{Y})},
$$

where

$$
\alpha(\boldsymbol{Y})=\mathrm{L}_{\boldsymbol{G}}^{n} \phi(\boldsymbol{Y}),
$$

and

$$
\beta(\boldsymbol{Y})=\mathrm{L}_{\boldsymbol{H}} \mathrm{L}_{\boldsymbol{G}}^{n-1} \phi(\boldsymbol{Y}) .
$$

In the second step, we apply a classical control strategy so that the system follows a reference in the transformed space. Suppose this reference, $\widetilde{\boldsymbol{X}}$, is such that 


$$
\dot{\tilde{X}}_{i}=\tilde{X}_{i+1}, \quad i=1, \ldots, n-1, \quad \dot{\tilde{X}}_{n}=\tilde{X}_{d},
$$

and define the error in the linear space as $e(t)=\tilde{X}_{1}-\tilde{Y}_{1}$. Then, the error dynamics is given by $e^{(n)}(t)=\tilde{X}_{d}-\tilde{u}$. A linear state feedback controller can then be used to stabilize this dynamics in the origin. This controller is given by

$$
\tilde{u}=\tilde{X}_{d}-\sum_{i=1}^{n} k_{i} e^{(i-1)},
$$

where the $k_{i}$ are positive constants. This leads to the stable error dynamics

$$
e^{(n)}+\sum_{i=1}^{n} k_{i} e^{(i-1)}=0
$$

and the controller's gains $k_{i}$ may be chosen to give the desired response. Controlled in this way, the slave system is able to follow any reference in the form of (14) restricted to the region $\Omega$. Since synchronization is desired, we use the master system as the reference. The transformation (6) is applied to the master system (1) yielding

$$
\widetilde{\boldsymbol{X}}_{d}=\Phi(\boldsymbol{X})
$$

and $\tilde{X}_{d}=\dot{X}_{n}$. As this reference signal is a trajectory of the master system transformed via a diffeomorphism, when the error $e(t)$ approaches zero the slave system will be synchronized with the master system.

As an example, we apply these ideas to the Lorenz system. The master system is taken as the usual equations

$$
\dot{x}_{1}=\sigma\left(y_{1}-x_{1}\right), \quad \dot{y}_{1}=r x_{1}-y_{1}-x_{1} z_{1}, \quad \dot{z}_{1}=-b z_{1}+x_{1} y_{1},
$$

and we suppose the parameter $b$ is unknown. The slave system is a copy of (18) but with this parameter treated as an input

$$
\dot{x}_{2}=\sigma\left(y_{2}-x_{2}\right), \quad \dot{y}_{2}=r x_{2}-y_{2}-x_{2} z_{2}, \quad \dot{z}_{2}=-u z_{2}+x_{2} y_{2} .
$$

In order to design the controller, we need to check if the system (19) is controllable. The system is put in the form of (2) leading to

$$
\boldsymbol{G}(\boldsymbol{Y})=[\sigma(y-x), r x-y-x z, x y]^{\mathrm{T}}
$$

and

$$
\boldsymbol{H}(\boldsymbol{Y})=[0,0,-z]^{\mathrm{T}} .
$$

Here the subscripts were dropped for clarity. As the first condition, the vector fields $\left\{\boldsymbol{H}, \operatorname{ad}_{\boldsymbol{G}} \boldsymbol{H}, \mathrm{ad}_{\boldsymbol{G}}^{2} \boldsymbol{H}\right\}$ must be linearly independent. Computing the Lie bracket of $\boldsymbol{G}$ and $\boldsymbol{H}$ leads to

$$
[\boldsymbol{G}, \boldsymbol{H}]=[0-x z-x y]^{\mathrm{T}} \text {. }
$$

Repeating the procedure we obtain

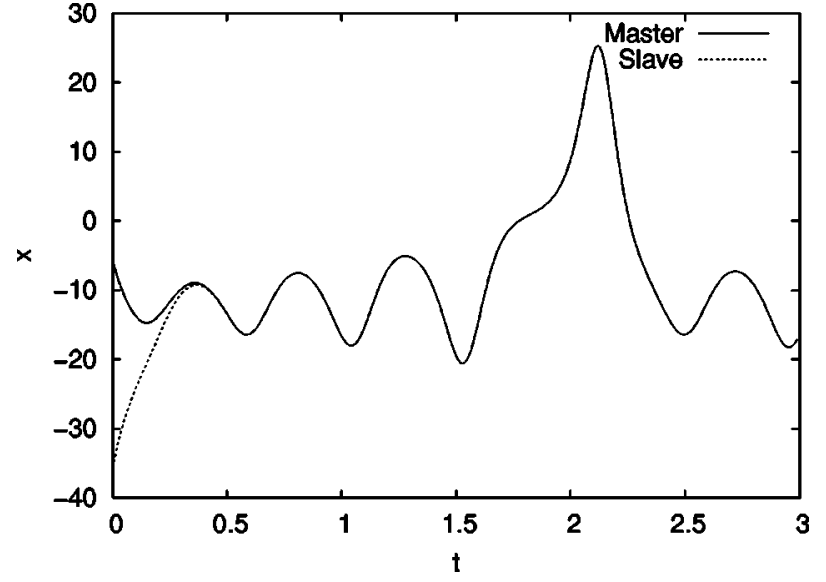

FIG. 2. The plot of $x$ in time for both master and slave systems. All quantities are dimensionless.

$$
[\boldsymbol{G},[\boldsymbol{G}, \boldsymbol{H}]]=\left[\begin{array}{c}
\sigma x z \\
(\sigma-1) x z-\sigma y z-2 x^{2} y \\
(\sigma+1) x y+2 x^{2} z-\sigma y^{2}-r x^{2}
\end{array}\right] .
$$

These vector fields are linearly independent if $\sigma x z \neq 0$. The second required condition is that the set $\left\{\boldsymbol{H}, \operatorname{ad}_{\boldsymbol{G}} \boldsymbol{H}\right\}$ must be involutive. To check this we must write $\left[\boldsymbol{H}, \operatorname{ad}_{\boldsymbol{G}} \boldsymbol{H}\right]$ as a linear combination of $\boldsymbol{H}$ and $\operatorname{ad}_{\boldsymbol{G}} \boldsymbol{H}$. The Lie bracket of $\boldsymbol{H}$ and $\operatorname{ad}_{\boldsymbol{G}} \boldsymbol{H}$ is given by

$$
\left[\boldsymbol{H}, \operatorname{ad}_{\boldsymbol{G}} \boldsymbol{H}\right]=\left[\begin{array}{lll}
0 & x z & -x y
\end{array}\right]^{\mathrm{T}} .
$$

By defining $c_{1}(\boldsymbol{Y})=2 x y / z$ and $c_{2}(\boldsymbol{Y})=-1,\left[\boldsymbol{H}, \operatorname{ad}_{G} \boldsymbol{H}\right]$ can be written as

$$
\left[\boldsymbol{H}, \operatorname{ad}_{\boldsymbol{G}} \boldsymbol{H}\right]=c_{1}(\boldsymbol{Y}) \boldsymbol{H}+c_{2}(\boldsymbol{Y}) \operatorname{ad}_{\boldsymbol{G}} \boldsymbol{H} .
$$

Therefore the set $\left\{\boldsymbol{H}, \operatorname{ad}_{\boldsymbol{G}} \boldsymbol{H}\right\}$ is involutive in the whole $\mathrm{R}^{3}$. This shows that the slave system given by (19) is controllable everywhere in $\mathbb{R}^{3}$ but in the planes $x z=0$.

The function $\phi(\boldsymbol{Y})$ needed to compute the diffeomorphism (9) is a solution to the following system of partial differential equation

$$
\begin{gathered}
\frac{\partial \phi}{\partial z} z=0 \\
\frac{\partial \phi}{\partial y} x z+\frac{\partial \phi}{\partial z} x y=0 \\
-\frac{\partial \phi}{\partial x} \sigma x z+\frac{\partial \phi}{\partial y}\left[(\sigma-1) x z-\sigma y z-2 x^{2} y\right] \\
+\frac{\partial \phi}{\partial z}\left[(\sigma+1) x y+2 x^{2} z-\sigma y^{2}-r x^{2}\right] \neq 0
\end{gathered}
$$

A simple solution to this system is $\phi(Y)=[x, 0,0]^{\mathrm{T}}$ which leads to the global diffeomorphism

$$
\tilde{\boldsymbol{Y}}=\Phi(\boldsymbol{Y})=\left[\begin{array}{c}
x \\
\sigma(y-x) \\
\left(\sigma r+\sigma^{2}\right) x-\left(\sigma+\sigma^{2}\right) y-\sigma x z
\end{array}\right] .
$$




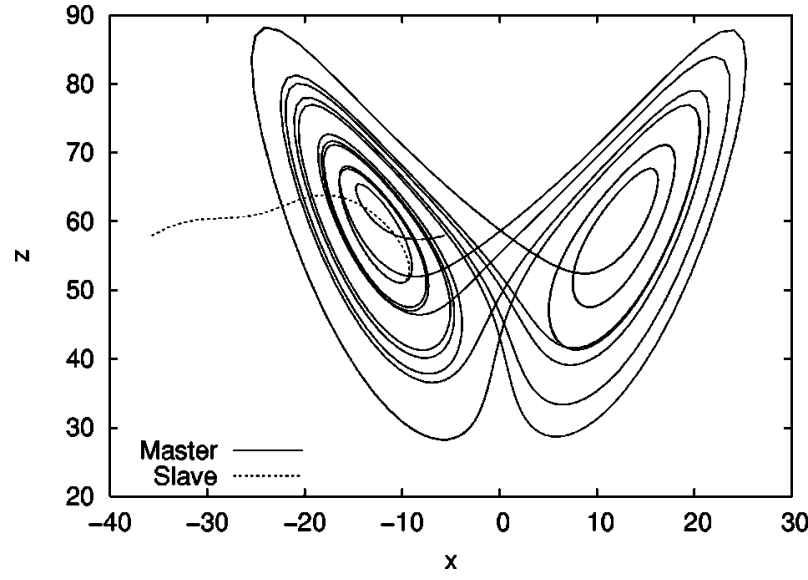

FIG. 3. The projections in the plane $x z$ of master's and slave's attractors. All quantities are dimensionless.

This transformation is applied to the slave system giving the transformed state coordinates $\tilde{\boldsymbol{Y}}$, and to the master system giving the reference $\tilde{\boldsymbol{X}}$. The value of $\tilde{\boldsymbol{X}}_{d}$ is obtained differentiating the last row of (27) which leads to

$$
\begin{aligned}
\tilde{\boldsymbol{X}}_{d}= & \left(\sigma r+\sigma^{2}\right) \sigma\left(y_{1}-x_{1}\right)-\left(\sigma+\sigma^{2}\right)\left(r x_{1}-y_{1}-x_{1} z_{1}\right) \\
& -\sigma\left[\sigma\left(y_{1}-x_{1}\right) z_{z}+x_{1}\left(-b z_{1}+x_{1} y_{1}\right)\right] .
\end{aligned}
$$

The controller used is of the form of (15), with the gains chosen as to give a fast nonoscillating response. The values chosen were $k_{0}=8000, k_{1}=1200$, and $k_{2}=60$.

The last step in the controller design is the inverse input transformation $\Psi^{-1}$. This transformation relates the control output in the linear space $\widetilde{u}$ to its counterpart in the original space $u$. The inverse transformation is given by (11). To compute it we need the functions $\alpha(\boldsymbol{Y})$ and $\beta(\boldsymbol{Y})$ given by

$$
\begin{aligned}
\alpha(\boldsymbol{Y})= & {\left[\left(\sigma r+\sigma^{2}\right)-\sigma z\right] \sigma(y-x)-\left(\sigma+\sigma^{2}\right)(r x-y-x z) } \\
& -\sigma x^{2} y
\end{aligned}
$$

and $\beta(\boldsymbol{Y})=\sigma x z$, respectively. Figure 2 shows our results for a Lorenz system with $(r, \sigma, b)=\left(60,10, \frac{8}{3}\right)$, with the $x$ state variable as a function of time for both the master and slave

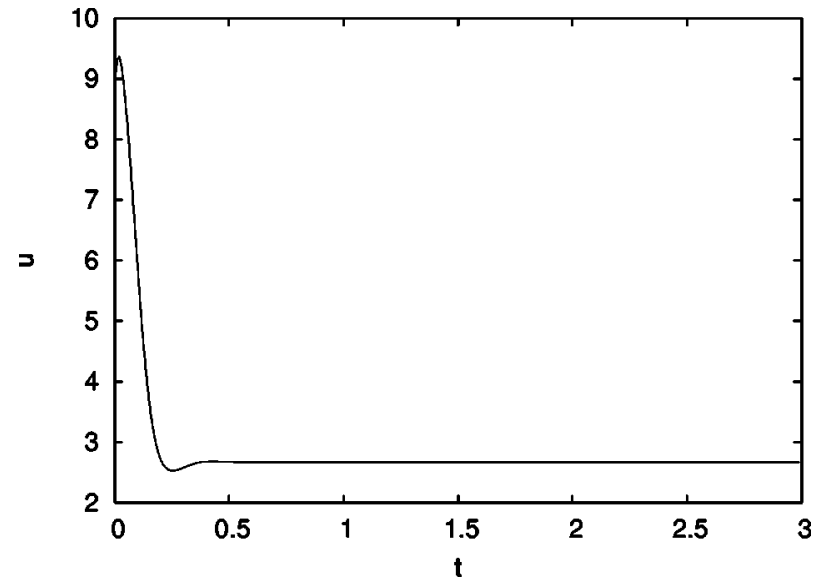

FIG. 4. The control action $u$ plotted against time showing convergence to the value of $b\left(\frac{8}{3}\right)$. All quantities are dimensionless.

systems. In this figure and in those that follow, all quantities plotted are dimensionless. A plane projection of both attractors is shown in Fig. 3. Synchronization is achieved as expected.

As a byproduct, the control action $u$ converges to the value of the unknown parameter $b$. This result is shown in Fig. 4

In summary, we presented a parameter estimation procedure for nonlinear systems that is based on synchronization and geometric control theory. Such theory allowed us to derive sufficient conditions for synchronization and parameter recovery. We used this technique to successfully estimate an unknown parameter in Lorenz's system.

The proposed method, however, still needs some improvements. First, any practical application involves noisy data, and the method must be modified to deal with this condition. Such modification is under conclusion and will be published in a future paper. Second, the proposed strategy needs the entire state vector $\boldsymbol{X}$ as input to the controller. This restricts the technique to applications where master's state vector can be fully measured. Hopefully this can be relaxed in the future, maybe via the use of geometric control theory.

The authors would like to acknowledge the support of FAPESP.
[1] H. Fujsaka and T. Yamada, Prog. Theor. Phys. 69, 32 (1983).

[2] L. M. Pecora and T. L. Carroll, Phys. Rev. Lett. 64, 821 (1990).

[3] A. Pikovsky, M. Rosenblum, and J. Kurths, Synchronization: A Universal Concept in Nonlinear Sciences (Cambridge University Press, Cambridge, 2001).

[4] G. Izus, P. Colet, M. San Miguel, and M. Santagiustina, Phys. Rev. E 68, 036201 (2003).

[5] X. F. Liao, X. M. Li, J. Pen, and G. R. Chen, Int. J. Commun. Syst. 17, 437 (2004).

[6] L. Zhao and E. E. N. Macau, IEEE Trans. Neural Netw. 12, 1375 (2001).

[7] E. Barreto, P. So, B. J. Gluckman, and S. J. Schiff, Phys. Rev. Lett. 84, 1689 (2000).
[8] D. B. Huang and R. W. Guo, Chaos 14, 152 (2004).

[9] A. Maybhate and R. E. Amritkar, Phys. Rev. E 59, 284 (1999).

[10] U. Parlitz, L. Junge, and L. Kocarev, Phys. Rev. E 54, 6253 (1996).

[11] A. Isidori, Nonlinear Control Systems (Springer, New York, 1995).

[12] G. Solis-Perales, V. Ayala, W. Kliemann, and R. Femat, Chaos 13, 495 (2003).

[13] H. Nijmeijer, Physica D 154, 219 (2001).

[14] F. M. M. Kakmeni, S. Bowong, C. Tchawoua, and E. Kaptouom, Phys. Lett. A 322, 305 (2004).

[15] J.-J. E. Slotine and W. Li, Applied Nonlinear Control (Prentice Hall, Englewood Cliffs, NJ, 1991). 